Results We describe novel and selective small molecule nonoligonucleotide TLR7/8 antagonists for the treatment of SLE. They exhibit potent activity in vitro in TLR-specific reporter systems $\left(\mathrm{IC}_{50}\right.$ of $\left.\sim 100 \mathrm{nM}\right)$ and in primary human blood cells $\left(\mathrm{IC}_{50}\right.$ of $50-500 \mathrm{nM}$ across various ligands and cytokine readouts), suppressing TLR7 and TLR8 but with no activity against TLR9 or other TLRs tested. Exploration of mechanism of action shows direct interaction of the lead compound with the external domain of TLR8. The compounds are orally available and active in a mouse model of R848 challenge. When tested in long-term dosing in pristane-induced or spontaneous NZB/W disease the compounds slow the advance of autoantibody titers and efficiently suppress development of nephritis and associated proteinuria.

Conclusions We have identified novel small molecule antagonists of human TLR7 and TLR8 with beneficial activity in mouse models of systemic lupus.

\section{IXAZOMIB, AN ORAL PROTEASOME INHIBITOR, DEPLETES PLASMA CELLS REDUCING AUTOANTIBODIES AND PDCS IN PRE-CLINICAL MODEL OF SYSTEMIC LUPUS ERYTHEMATOSUS}

${ }^{1} Y$ Itomi* ${ }^{1} \mathrm{~T}$ Tanaka, ${ }^{1} \mathrm{M}$ Sagara, ${ }^{2} \mathrm{~T}$ Kawamura, ${ }^{1} \mathrm{~T}$ Sato. ${ }^{1}$ Takeda Pharmaceutical Company, Immunology Unit, Fujisawa- Kanagawa, Japan; ${ }^{2}$ Takeda Pharmaceutical Company, Regenerative Medicine Unit, Fujisawa- Kanagawa, Japan

\subsection{6/lupus-2017-000215.88}

Background and aims Auto-antibodies to nuclear constituents and type I Interferons (IFN) such as IFN-a play key roles in pathogenesis of Systemic Lupus Erythematosus (SLE). Ixazomib, an oral proteasome inhibitor, approved in the US and Canada for use in combination with lenalidomide and dexamethasone in patients with multiple myeloma who have received at least 1 prior therapy. Proteasome inhibitors like ixazomib that may deplete plasma cells and cellular sources of IFN-a are also attractive for autoimmune diseases like SLE. To investigate the potential of ixazomib the MRL/lpr model was used as it has extensively been shown to replicate many features of SLE.

Methods MRL/lpr animals received oral ixazomib twice a week for 4 weeks.

Results Ixazomib suppressed the time-dependent increase in anti-dsDNA IgG antibodies, resulting in $73 \%(\mathrm{p}<0.01)$ inhibition of autoantibodies at the end of treatment versus vehicle. In ELISpot assays, ixazomib decreased the number of antidsDNA IgG antibody-secreting cells in spleen by $25 \%$ $(\mathrm{p}<0.01)$. In addition, FACS analysis revealed that ixazomib decreased both splenic plasma cells by $39 \%(p<0.001)$ and plasmacytoid dendritic cells (pDCs) by 38\% (p<0.01), with treatment.

Conclusions These findings suggest that ixazomib may be an effective agent for treating antibody-mediated diseases such as SLE by depleting both plasma cells the source of pathogenic antibodies and pDCs the main source of type I IFN production.

An ongoing randomised, double-blind phase Ib study is investigating multiple rising doses of Ixazomib (MLN9708) for the treatment of patients with ISN/RPS class III, IV or $\mathrm{V}$ lupus nephritis who have not responded adequately to current therapy.

\section{TLR7 AND TLR8 TARGETED MICRO-RNAS INHIBIT SIGNALLING AND SUPPRESS INFLAMMATION IN A NOVEL HUMAN-MOUSE CHIMERIC MODEL OF SYSTEMIC LUPUS ERYTHEMATOSUS}

N Young, G Valiente, H Steigelman, J Hampton, W Jarjour*. The Ohio State University Wexner Medical Centre, Division of Rheumatology and Immunology, Columbus, USA

\subsection{6/lupus-2017-000215.89}

Background and aims We have previously demonstrated that toll-like receptor (TLR)7 and TLR8 are significantly up-regulated in peripheral blood mononuclear cells (PBMCs) of systemic lupus erythematosus (SLE) patients and can be further induced with oestrogen treatment. It has recently been shown that specific micro-RNA (miR) sequences packaged in extracellular vesicles can stimulate these receptors in addition to the conventional activation by binding single-stranded RNA of viral origin. The aim of this study was to explore the feasibility of using miR antagonists to block TLR7 and TLR8-mediated inflammatory pathways.

Methods Human-mouse chimaeras were generated by adoptively transferring PBMCs from active SLE patients into immunodeficient NOD-scid IL-2r $\gamma$ (null) mice using a modified protocol that we previously established in Sjöjren's syndrome. Prior to transfer, SLE patient PBMCs were treated either with a cocktail of locked nucleic acid antagonists targeting several miRs or nonsense, scrambled controls. At 21 days post-transfer, blood was collected for flow cytometry and cytokine analysis; tissues were processed for histopathological examination by $\mathrm{H}$ and $\mathrm{E}$ and immunohistochemistry.

Results The phenotypic characteristics of various immune cells were similar in both experimental groups; however, inhibition with miR antagonists reduced levels of human IL-2, IL-6, IL10 , and TNF- $\alpha$ relative to scramble (control) treatment. Histopathological analysis revealed that $\mathrm{miR}$ antogonists inhibited the robust responses detected with control treatment in the small intestine, liver, and kidney. Further characterisation of infiltrates confirmed the presence human CD3 + T-cells.

Conclusions These data establish a novel model to study SLE and provide experimental evidence that TLR7 and TLR8 targeted miR antagonists have therapeutic potential in SLE.

\section{SAFETY, EFFICACY AND TRANSCRIPTIONAL CHANGES FOLLOWING REPEATED ADMINISTRATION OF DAPIROLIZUMAB PEGOL IN PATIENTS WITH SYSTEMIC LUPUS ERYTHEMATOSUS: RESULTS FROM A PHASE I STUDY}

${ }^{1} \mathrm{C}$ Chamberlain, ${ }^{2} \mathrm{P}$ Colman, ${ }^{3} \mathrm{~A}$ Ranger, ${ }^{4} \mathrm{G}$ Johnson* ${ }^{5} \mathrm{C}$ Otoul, ${ }^{6} \mathrm{C}$ Stach, ${ }^{7} \mathrm{~T}$ Dörner, ${ }^{8} \mathrm{M}$ Urowitz, ${ }^{7} \mathrm{~F}$ Hiepe. ${ }^{7} \mathrm{U} C B$, Experimental Medicine and Diagnostics, Slough, UK; ${ }^{2} U C B$ Pharma, Exploratory Statistics, Slough, UK; ${ }^{3}$ Biogen, Translational Medicine and Biomarkers, Cambridge, USA; ${ }^{4}$ UCB Pharma, Clinical Biomarkers and Experimental Therapeutics, Slough, UK; ${ }^{5}$ UCB Pharma, Exploratory Statistics, Braine-L'Alleud, Belgium; ${ }^{6}$ UCB Pharma, Physician Development and Excellence, Braine-L'Alleud, Belgium; ${ }^{7}$ Charite Universitätsmedizin Berlin, Department of Medicine/Rheumatology and Clinical Immunology, Berlin, Germany; ${ }^{8}$ University of Toronto, Centre for Prognosis Studies in the Rheumatic Diseases, Toronto, Canada

\subsection{6/lupus-2017-000215.90}

Background and aims Binding of CD40 ligand (CD40L) to CD40 activates B cells, antigen-presenting cells and platelets. Evidence suggests CD40L blockade might provide an effective treatment for systemic autoimmune disorders, including 